\title{
PERCEPCIÓN DEL LIDERAZGO EN LAS(OS) ENFERMERAS(OS) DE UN HOSPITAL DEL SUR DE CHILE ${ }^{1}$
}

\section{LEADERSHIP PERCEPTION IN NURSES BELONGING TO A SOUTHERN HOSPITAL IN CHILE}

\author{
Cyntia Quezada Torres* \\ Mónica Illesca Pretty ${ }^{* *}$ \\ Mirtha Cabezas GonZÁlez ${ }^{* * *}$
}

\begin{abstract}
RESUMEN
Objetivo: Conocer la percepción del Liderazgo en las(os) Enfermeras(os) de un Hospital del Sur de Chile. Método: Se realizó una investigación cualitativa, descriptiva, exploratoria e interpretativa a través de un estudio de caso único. Los informantes claves fueron seis Enfermeras(os) que se desempeñan en puestos de responsabilidad. La recolección de datos fue mediante grupo focal. El análisis siguió un esquema a través de la reducción progresiva (separación de unidades, agrupamiento, identificación y clasificación de elementos), disposición, transformación y obtención de conclusiones verificables, validándose a través de la triangulación por investigadores. Resultados: Del discurso surgieron 118 unidades de significado, emergiendo 4 categorías. Las de mayor frecuencia fueron "Significado del Liderazgo" (40), "Liderazgo en las funciones" (33), y "Factores que facilitan el desarrollo del Liderazgo" (28). En tanto la de menor frecuencia fue "Factores que obstaculizan el desarrollo del Liderazgo" (17). Al final del proceso se levantan dos dominios cualitativos "Conceptualización del Liderazgo que tienen las(os) Enfermeras(os) de un Hospital del sur de Chile" y "Correspondencia entre las funciones de Enfermería y el ejercicio del Liderazgo". Conclusión: El Liderazgo es percibido por la mayoría como un conjunto de características y no como un proceso de influencia; no existe un concepto claro del mismo, sin embargo se reconoce como un elemento fundamental para las distintas funciones en el ámbito de la práctica profesional, primando en el asistencial, donde se identifican en general más factores facilitadores que obstaculizadores y asociados a la "Calidad de atención del paciente".
\end{abstract}

Palabras clave: Liderazgo, enfermería, competencia profesional.

\begin{abstract}
Objective: To know the perception of Nurses Leadership (os) at San Carlos Hospital, from Ancud city in Chiloe island, Chile. Method: A qualitative, descriptive, exploratory and interpretative through a single case study, was conducted. Key informants were six nurses working in responsibility positions. The data were collected through focus group. The analysis followed a pattern through the progressive reduction (separation units, grouping, identification and classification of elements), disposal, processing and obtaining verifiable conclusions, validating it through triangulation by researchers. Results: From the discourse emerged 118 units of meaning, emerging four categories. The most frequent ones were "Meaning of Leadership" (40), "Leadership in the functions" (33), and "Factors facilitating leadership development" (28). While the lowest frequency one was "Factors that hinder

\footnotetext{
${ }^{1}$ El contenido de este artículo es parte de la tesis desarrollada por la autora para optar al grado de Magíster en Enfermería, mención Gestión del Cuidado, Universidad de La Frontera.

*Enfermera, Hospital San Carlos, Ancud. Chile. E-mail: cyntiaquezada@hotmail.com

${ }^{* *}$ Enfermera. Académico Enfermera Medicina Interna. Universidad de La Frontera. Facultad de Medicina. Manuel Montt 112 Temuco, Chile. E-mail: monica.illesca@ufrontera.cl

${ }^{* * *}$ Enfermera. Académico Ciencias Preclínicas. Universidad de La Frontera Facultad de Medicina. Manuel Montt 112 Temuco, Chile.E-mail: mirtha.cabezas@ufrontera.cl
} 
leadership development" (17). At the end of the two qualitative domains rise "Conceptualizing Leadership with nurses (I) at San Carlos Hospital (Ancud)" and "Correspondence between functions and Nursing Leadership exercise". Conclusion: Leadership is perceived by most as a set of features and not as a process of influence; there is no clear concept of it, but it is recognized as a fundamental element for the different functions within the scope of professional practice, focusing on care, which identifies more generally hindering and facilitating factors associated with the "Quality of patient care".

Key words: Leadership, nursing, competence professional.

Fecha recepción: 21/11/12. Fecha aceptación: 17/03/14.

\section{INTRODUCCIÓN}

En materia de gestión, las(os) enfermeras(os) tienen gran participación en el logro de los objetivos, asumiendo un rol de líder, en el cual deben realizar proyectos para entregar respuestas en función de ellos, influyendo sobre otros integrantes del equipo para una efectiva participación (1). Esto representa un desafío importante para la disciplina, ya que contribuye al desarrollo de los Servicios de Salud en forma integral, eficiente, equitativa y de calidad para lograr salud para todos (2).

De lo anterior se deduce la importancia del Liderazgo en las Instituciones de Salud (3). En este sentido se acepta que es un fenómeno que condiciona las funciones desarrolladas por las(os) enfermeras(os) en el ejercicio del cuidado de las personas e influirá en el cómo se expresa en el trabajo diario de estos profesionales; ayuda en la coordinación del personal de salud, al enfrentar conflictos $\mathrm{y}$ tomar decisiones (4). Permite guiar las discusiones del equipo sobre la práctica y fortalecer los cambios en las rutinas (5). El rol es considerado una de las competencias básicas que se realiza durante el trabajo diario, por lo que debería ser reconocida en la administración de los Establecimientos de Salud) (6).

No obstante, existe evidencia de la falta de preparación de ellos en Liderazgo durante el trabajo diario con el personal de salud. Esta inexperiencia se asocia a un déficit de formación profesional, ya que esta última está más enfocada a la educación en gestión, pese a que reconocen la estrecha vinculación de esta competencia con el desarrollo de sus funciones. Al respecto, la teoría sociológica respalda un Liderazgo que se puede cultivar, siendo un proceso continuo de aprendizaje, y es esencial la valoración que estos profesionales hagan de él para aplicarlo en su actividad diaria (7).

Ante los cambios políticos, sociales, epidemiológicos y económicos en América Latina, la disciplina de Enfermería ha tenido una mirada vanguardista que le ha permitido permanecer vigente, respetada y con un Liderazgo reconocido, por responder a las necesidades de salud y al bienestar del ser humano, a través de la Gestión del Cuidado, con un enfoque holístico, ético e interpersonal (8). En este ámbito la American Organization of Nurse Executives (AONE) ha reconocido el Liderazgo como una competencia importante en las(os) Enfermeras(os) (9).

Por su participación activa en la formulación de políticas públicas, programas sanitarios, asignación de recursos, análisis de información y dirección de grupos de trabajos en las funciones asistencial, de educación, administración e investigación, la Organización Mundial de la Salud (OMS) ha señalado: "las Enfermeras(os) que ocupan posiciones de Liderazgo deben ser capaces de influir en los mecanismos de adopción de decisiones que establecen prioridades y asignan recursos para la obtención de salud" (2).

Del mismo modo, Malone plantea que "el Liderazgo es la competencia esencial de las Enfermeras(os) de prácticas avanzadas" 
(10), con el objetivo de mejorar la salud de la sociedad, aplican un conocimiento integrado, se anticipan a los cambios con visión de futuro, con un deseo de otorgar poder a la profesión ejerciéndola en base a los valores de la disciplina (10). En consecuencia, aquellas(os) que tienen cargos de responsabilidad en las Instituciones de Salud deben desempeñar las funciones de dirigir, motivar y supervisar a su equipo, en base a las metas del Establecimiento e influyendo así en el comportamiento de éstos (11).

El Liderazgo es un constructo complejo y multideterminado, el cual se ha investigado desde hace muchos años y es definido como "un proceso natural que ocurre entre una persona Líder y sus seguidores, existiendo consenso que éste es necesario para guiar a las organizaciones hacia objetivos estratégicos" (12). El líder, a través de sus propias acciones, debe crear una cultura, donde el cambio no sea considerado una amenaza, sino una posibilidad de crecimiento y desarrollo para los integrantes, asegurando el futuro de la Institución (13).

Existen diversas definiciones de Liderazgo, entre ellas: "relación de individuos, en la que se da una influencia de una persona sobre un grupo para conducirlo hacia determinadas metas" (14); "fenómeno de influencia social, es la suma de los esfuerzos individuales para alcanzar los objetivos compartidos de un grupo" (4) y "fenómeno de grupo donde se presenta un sistema de influencia social de un individuo sobre los demás, y es un proceso colectivo para compartir entre sus integrantes" (15).

El Proyecto de Liderazgo para el Cambio Fase II, del Consejo Internacional de Enfermeras, sostiene que se debe tener "una actitud proactiva, visionaria y asertiva hacia los procesos de la Reforma" (16), es decir tener un rol protagónico como líder, ampliar su horizonte, con un enfoque multisectorial y multidisciplinario, crear redes de trabajo, mostrar el valor de la disciplina y conducir los cambios con la gestión oportuna de los procesos.

En Chile, a partir de la Reforma en Salud se establece una nueva Autoridad Sanitaria (16), otorgando a las(os) Enfermeras(os) la responsabilidad de la "Gestión de los Cuidados de las personas", lo que también se instituye en la Ley $\mathrm{N}^{\circ} 19.536$ (17), Artículo 113 del Código Sanitario (18). El legislador asigna tres nuevas funciones: "Gestión del Cuidado, el ejecutar acciones derivadas del diagnóstico y tratamiento médico; y el deber de velar por la mejor administración de recursos de asistencia para el paciente" (19). En consecuencia, el Liderazgo se expresa cuando se le encomienda a las(os) Enfermeras(os) velar por la mejor administración de los recursos asistenciales, asumiendo un papel garante en la asistencia y derechos de los pacientes (20).

A través de la observación empírica, en un Hospital del sur de Chile se observa en las(os) Enfermeras(os) pocas acciones que reflejen el Liderazgo como una competencia. Se muestra un déficit en la conducción del personal, falta de claridad al entregar información, escasa empatía-carisma, inexistencia de análisis de situaciones y toma de decisiones, lo que conlleva a conflictos con el equipo, sin logros en la planificación del trabajo en los servicios. Lo anterior puede deberse a factores, tales como: presencia de profesionales jóvenes, inexperiencia, dificultades de tipo relacional, objetivos no claros, ausencia de confianza y de apoyo de la autoridad, lo que repercute claramente en la atención directa de los usuarios.

Asimismo, existe la percepción de no ser consideradas(os) y enfrentar factores externos del medio que obstaculizan su desarrollo, como los problemas de relaciones con médicos, sindicatos, autoridades, personal de salud. Se visualiza un ambiente conflictivo donde no se reconoce a Enfermería como disciplina, lo que origina condiciones adversas para desarrollar un efectivo liderazgo.

Con el propósito de contribuir al fortalecimiento del liderazgo en el ejercicio de la profesión, se realizó una investigación cua- 
litativa exploratoria, descriptiva e interpretativa a través de un estudio de caso único, donde los informantes claves fueron seis enfermeras(os) que se desempeñan en puestos de responsabilidad. El objetivo del estudio fue conocer la Percepción del Liderazgo en las(os) Enfermeras(os) que desarrollan puestos de responsabilidad y cumplen funciones en los cuidados de los pacientes en un Hospital del sur de Chile, durante el primer semestre de 2011. Los objetivos específicos se orientaron a: develar el significado de Liderazgo, explorar los factores facilitadores y obstaculizadores en el ejercicio del Liderazgo e indagar sobre el ejercicio del Liderazgo en las funciones asistencial, administrativa, de docencia e investigación.

Realizar este tipo de investigación provee información en dos ámbitos: fortalece la corriente cualitativa en temas de gestión y apoya al desempeño de las(os) Enfermeras(os), que se esfuerzan por liderar equipos de trabajo en la Gestión del Cuidado, promoviendo la participación, comunicación y cohesión grupal (13).

\section{MATERIAL Y MÉTODO}

Investigación sustentada bajo el paradigma cualitativo, desde un enfoque exploratorio, descriptivo e interpretativo, a través de un estudio de caso único. La muestra fue intencionada de casos por criterios, conformada por seis enfermeras(os), con criterios de inclusión de trabajar en el Hospital San Carlos de Ancud-Chiloé, con desempeño en el ámbito de la Gestión del Cuidado, que asumen Jefaturas de Servicios, y a la vez funciones asistenciales y administrativas, y que aceptaron firmar previamente Formulario Consentimiento Informado. Se encontraban en calidad de contrata y titular, con tiempo de antigüedad desde 2 a 20 años, con distintas responsabilidades, entre ellas ejercer Liderazgo. La recolección de datos se realizó a través de un grupo focal, en una sesión de una hora y media, en el Auditórium del Establecimiento, en un ambiente favorable, previa aplicación de una pauta semiestructurada, donde sus integrantes expresaron sus opiniones de manera libre en relación al problema que se investiga. Se consideró la participación de tres personas: una para registrar las notas de campo, otra para grabar la información y la investigadora principal quien guió la técnica, dando el inicio con el enunciado de la pregunta orientadora. Los aspectos éticos en esta investigación fueron resguardados por el Consentimiento Informado de los informantes claves (21) y autorización del director del Servicio de Salud, quien actuó como representante del Comité de Ética.

El análisis de datos fue el de comparaciones constantes (22), no agrupándose en categorías predeterminadas, sino más bien emergiendo de un proceso de razonamiento inductivo. Se adoptó el esquema de la reducción progresiva de la información concordante con la interrogante del estudio (separación de unidades, agrupamiento, identificación y clasificación de elementos), disposición, transformación y obtención de conclusiones verificables (23), utilizando la metodología propuesta por Van Maanen (24):

- Nivel 1: Identificación y segmentación de narraciones textuales para agrupar en categorías descriptivas (primera aproximación a núcleos temáticos o emergentes).

- Nivel 2: Dominios cualitativos a través de la identificación de similitudes estructurales y elementos comunes de las categorías del Nivel 1.

- Nivel 3: Identificación de dominios cualitativos (análisis secuencial y transversal de las metacategorías).

La rigurosidad científica se garantizó utilizando criterios de rigor: credibilidad (triangulación por investigador: observación por 
más de una persona del mismo fenómeno o situación), dependencia (réplica paso a paso, permitiendo juzgar el grado de correspondencia con otros contextos), confirmabilidad (ejercicio de reflexión y consenso con otros investigadores) y transferibilidad (recogida abundante información descriptiva hasta la saturación de los datos y desarrollo de descripciones minuciosas) (25).

\section{RESULTADOS}

El análisis de los datos derivados del grupo focal siguió un esquema que en forma pro- gresiva generó una reducción de la información concordante con las interrogantes del estudio, a través de tres niveles:

Nivel 1: se encontraron 118 unidades de significado relevantes para el estudio agrupado en 4 categorías descriptivas emergentes. En la Tabla 1 se presenta la distribución de frecuencias de las unidades de significado de cada una de las categorías codificadas y analizadas, en función de la percepción de las(os) Enfermeras(os) de un Hospital del sur de Chile en relación al Liderazgo.

Nivel 2: representa los núcleos temáticos emergentes o metacategorías que surgen del

Tabla 1. Distribución de frecuencias de las unidades de significado de las categorías descriptivas codificadas con respecto al objeto de estudio.

\begin{tabular}{|c|c|c|c|}
\hline $\begin{array}{l}\text { Categorías } \\
\text { descriptivas } \\
\text { codificadas }\end{array}$ & & ades de significado & $\mathrm{n}$ \\
\hline \multirow{10}{*}{$\begin{array}{l}\text { "Significado } \\
\text { del Liderazgo" } \\
\text { (SLI) }\end{array}$} & \multirow{7}{*}{ Características del Líder (CLI) } & Credibilidad & 8 \\
\hline & & Capacidad para trabajo en equipo & 7 \\
\hline & & Trascendencia & 4 \\
\hline & & Respetado & 4 \\
\hline & & Capacidad establecer metas y objetivos & 4 \\
\hline & & Personalidad & 3 \\
\hline & & Empatía & 2 \\
\hline & \multirow{3}{*}{ Bases del liderazgo (BLI) } & Jerarquía en el grupo y en la Institución & 7 \\
\hline & & Competencias & 1 \\
\hline & & Total categoría & 40 \\
\hline \multirow{9}{*}{$\begin{array}{l}\text { "Liderazgo en } \\
\text { las funciones" } \\
\text { (LFUN) }\end{array}$} & \multirow[t]{3}{*}{ Función Asistencial (FASI) } & Calidad de atención & 11 \\
\hline & & Trabajo en equipo & 4 \\
\hline & & Toma decisiones & 3 \\
\hline & Función Educación (FEDU ) & Muy poco liderazgo & 2 \\
\hline & Función de investigación (FINV) & Nada de liderazgo & 2 \\
\hline & \multirow{4}{*}{ Función Administrativa (FADM) } & Gestión & 4 \\
\hline & & Toma decisiones y manejo de conflictos & 4 \\
\hline & & Formula objetivos & 3 \\
\hline & & Total categoría & 33 \\
\hline
\end{tabular}




\begin{tabular}{|c|c|c|c|}
\hline \multirow{8}{*}{$\begin{array}{l}\text { "Factores que } \\
\text { facilitan el } \\
\text { desarrollo } \\
\text { del Liderazgo" } \\
\text { (FFDL) }\end{array}$} & \multirow{3}{*}{ Cualidades de Liderazgo (CLD) } & Conocimiento del Liderazgo & 7 \\
\hline & & Comunicación & 5 \\
\hline & & Experiencia & 4 \\
\hline & \multirow{2}{*}{ Estructura curricular (ECU) } & Formación de Enfermería y Centros de Prácticas & 5 \\
\hline & & Actualizaciones de Enfermería & 1 \\
\hline & \multirow{3}{*}{ Institucional (INST) } & Organización & 4 \\
\hline & & Prácticas laborales y reuniones & 2 \\
\hline & & Total categoría & 28 \\
\hline \multirow{9}{*}{$\begin{array}{l}\text { "Factores que } \\
\text { obstaculizan el } \\
\text { desarrollo del } \\
\text { Liderazgo" } \\
\text { (FODL) }\end{array}$} & \multirow{4}{*}{ Relación Médico Enfermera (RME) } & No reconocimiento a Enfermería & $\overline{4}$ \\
\hline & & Distancia Médico-Enfermera(o) & 3 \\
\hline & & Resistencia y temor a los Médicos & 3 \\
\hline & & Conducta negativa del Médico & 1 \\
\hline & $\underline{\text { Estructura Curricular (ECU) }}$ & Escasa formación en Liderazgo & 3 \\
\hline & Enfermería (ENF) & Cultura & 2 \\
\hline & \multirow[t]{3}{*}{ Relación Gremios Enfermería (RGE) } & Resistencia de los Gremios & 1 \\
\hline & & Total categoría & 17 \\
\hline & & Total & 118 \\
\hline
\end{tabular}

Tabla 2. Distribución porcentual entre metacategorías, categorías descriptivas codificadas y unidades de significado con respecto al objeto de estudio.

\begin{tabular}{llcc}
\hline Metacategorías & \multicolumn{1}{c}{ Categorías descriptivas codificadas } & \multicolumn{2}{c}{$\begin{array}{l}\text { Unidades de } \\
\text { significado }\end{array}$} \\
\cline { 2 - 4 } & & No & $\%$ \\
\hline Conocimiento del Liderazgo & "Significado del Liderazgo" (SLI) & 40 & 33,9 \\
\hline $\begin{array}{l}\text { Relación entre Liderazgo y las funciones } \\
\text { de Enfermería }\end{array}$ & "Liderazgo en las funciones" (LFUN) & 33 & 28 \\
\hline $\begin{array}{l}\text { Factores asociados al desarrollo del } \\
\text { Liderazgo }\end{array}$ & $\begin{array}{l}\text { "Factores que facilitan el desarrollo del } \\
\text { Liderazgo" (FFDL) } \\
\text { "Factores que obstaculizan el desarrollo } \\
\text { del Liderazgo" (FODL) }\end{array}$ & 28 & 23,7 \\
\hline & & 17 & 14,4 \\
\hline
\end{tabular}

nivel 1, las cuales se agruparon y estructuraron en 4 categorías (Tabla 2). Posterior a un proceso de comparación intercategorías en el que se buscaron similitudes estructurales y elementos comunes de este análisis, se identificaron 3 metacategorías en torno a los cuales se reagruparon todas las categorías.

\section{7} 5 4

$$
4
$$

\section{8}

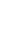
3 (3)

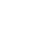
2 (1)

\section{8}


Tabla 3. Relación de los dominios cualitativos emergentes con sus respectivas metacategorías y categorías.

\begin{tabular}{|c|c|c|}
\hline Dominios & Metacategorías & Categorías \\
\hline $\begin{array}{l}\text { Dominio } 1 \text { "Conceptualización } \\
\text { del Liderazgo que tienen las(os) } \\
\text { Enfermeras(os) del Hospital San Carlos } \\
\text { de Ancud" }\end{array}$ & $\begin{array}{l}\text { Conocimiento de } \\
\text { Liderazgo }\end{array}$ & Significado del Liderazgo "SLI" \\
\hline \multirow[t]{3}{*}{$\begin{array}{l}\text { Dominio } 2 \text { "Correspondencia entre las } \\
\text { funciones de Enfermería y el ejercicio del } \\
\text { Liderazgo" }\end{array}$} & $\begin{array}{l}\text { Relación entre Liderazgo } \\
\text { y las funciones de } \\
\text { Enfermería }\end{array}$ & $\begin{array}{l}\text { Liderazgo en funciones Asistenciales, } \\
\text { Administrativas, Docencia e } \\
\text { Investigación "FUN" }\end{array}$ \\
\hline & $\begin{array}{c}\text { Factores asociados al } \\
\text { desarrollo del Liderazgo }\end{array}$ & $\begin{array}{c}\text { Factores que obstaculizan el } \\
\text { desarrollo del Liderazgo "FODL" }\end{array}$ \\
\hline & & $\begin{array}{c}\text { Factores que facilitan el desarrollo } \\
\text { del Liderazgo "FFDL" }\end{array}$ \\
\hline
\end{tabular}

\section{DISCUSIÓN Y CONCLUSIÓN}

Se destaca en la categoría "Significado del Liderazgo" dos temas: "Características del Líder" y "Bases del Liderazgo", lo que reveló que no existe una concepción de Liderazgo como competencia (26). Lo anterior orienta la mirada hacia las teorías iniciales de Liderazgo, la cual se centraba en los atributos, a diferencia de las actuales que se enfocan en el proceso de influencia, donde el líder tiene la capacidad de guiar a las personas hacia el logro de objetivos comunes (27).

Las(os) informantes claves conciben el Liderazgo con las "Características del Líder", valorando mayoritariamente las competencias genéricas: "Credibilidad" (20,0\%), "Capacidad para trabajo en equipo" (17,5\%), "Respetado por otros" (10,0\%), "Capacidad establecer metas y objetivos" (10,0\%), "Personalidad" (7,5\%) y "Empatía" (5.0\%). Asimismo, describen las bases de éste como la "Jerarquía en el grupo y en la Institución" $(7,0 \%)$, lo que evidencia un reconocimiento por el equipo dado por el cargo que ocupan en el establecimiento. Las "Competencias", pese a ser mencionadas en un porcentaje menor son esenciales. Su valoración independiente significa que en el ámbito de En- fermería es indispensable tener fortalezas manifiestas en este campo, el saber le otorga la propiedad de ejercerlo, más aún si se considera que para las organizaciones de salud, como producto de la globalización y el impacto tecnológico, requieren de profesionales eficientes, que otorguen servicios orientados al ser humano, basado en las competencias genéricas y disciplinares (28). Las cualidades identificadas son términos que coinciden con los resultados de otros estudios y definiciones del Liderazgo $(27,29-31)$, las cuales se pueden relacionar con el estilo de Liderazgo transformacional, coincidiendo con lo realizado en Colombia a 94 docentes del Programa de Enfermería, cuyo objetivo era establecer la relación de tipos de Liderazgo transformacional y transaccional (32), destacándose que el primero va en aumento y es el más utilizado en la práctica (33).

Los informantes claves otorgan importancia a la función "Asistencial" en lo que se refiere a "Calidad de Atención del paciente", destacando en los discursos "Orden" " "Cumplimiento de Normas". Este resultado refleja que la disciplina de Enfermería se preocupa del bienestar del ser humano, con una mirada integral, a través de la Gestión del Cuidado (8), debiendo tener competencias técnicas $y$ de gestión (34). En lo "Administrativo", la 
importancia de los atributos que emergen garantiza la continuidad de la atención, favoreciendo el trabajo en equipo y coordinación de los servicios (35). Insuficiente información se obtuvo en las áreas de "Educación" e "Investigación", pese a reconocer la importancia de ambas dentro del desarrollo de la profesión, existiendo pocas publicaciones que ratifiquen este hecho (36).

Los aspectos que dificultan el desarrollo del Liderazgo en las(os) Enfermeras(os) se relacionan con los grupos de poder existentes en los centros asistenciales (37), lo que permite señalar que en las Instituciones de Salud existen condiciones no propicias para el ejercicio del Liderazgo, repercutiendo directamente en los cuidados de los pacientes. Existen investigaciones que exponen que este entorno conflictivo se asocia a efectos adversos y mortalidad de los pacientes (38). Los factores facilitadores serían potenciados por las relaciones positivas y el respeto mutuo (39).

A su vez, el ejercicio del Liderazgo se ve facilitado por el "Conocimiento", reconociendo al líder eficaz, como aquel capacitado y competente (40) mediante una buena argumentación, firmeza en las decisiones y convicciones claras, son términos que coinciden con los resultados obtenidos en un investigación realizada a Enfermeras(os) de la Universidad Pontificia Bolivariana, donde plantean lo necesario de contar con profesionales de Enfermería que tengan competencias para ejercer con maestría este proceso en el ámbito donde se desempeñen (27). Otro elemento señalado es la "Capacidad de establecer Metas y Objetivos del grupo", reconocida su importancia en el mismo estudio. Esta cualidad también coincide con un trabajo realizado en Australia, cuyo propósito fue conocer el fenómeno del Liderazgo por seis Enfermeras de una Unidad de Cuidados Intensivos Adulto de un Hospital Público (29). Además se asocia a una de las definiciones de Liderazgo mostrada en otro estudio realizado en España (30).

$\mathrm{Al}$ igual que en el estudio realizado en
Lima, Perú (31), la "Credibilidad" (20,0\%) y la "Capacidad para trabajo en equipo" $(17,5)$ es parte de lo que las(os) Enfermeras(os) del Hospital San Carlos de Ancud esperan del líder.

También la "Experiencia" se constituye como un hecho fundamental en la adquisición del Liderazgo, lo que es reconocido por el Colegio Internacional de Enfermeras, para lo cual implementa programas de formación en distintos lugares del mundo (41).

Se puede concluir que no existe un concepto claro de Liderazgo, sin embargo se reconoce como un elemento fundamental en las distintas funciones en el ámbito de la práctica profesional. A pesar de no declararse como una competencia, las(os) informantes claves mencionan características genéricas que se asocian a ella. Identifican principalmente factores facilitadores como las cualidades del Liderazgo, enfocando sus comentarios a competencias genéricas, al proceso de formación de las(os) Enfermeras(os) y Centros de Prácticas y actualización en Enfermería. En tanto, alude la existencia de una serie de factores que les afectan seriamente su ejercicio, como lo es un ambiente conflictivo a pesar de reconocer Autonomía e Identidad propia. Entre los aspectos obstaculizadores más relevantes se devela el estamento médico, quienes no reconocen a Enfermería como disciplina, desvalorizándola, y generando un fuerte grupo de resistencia. Al respecto, existe la percepción de no contar con herramientas necesarias para revertir esta situación, refieren que no son tomadas en cuenta, sin opinión.

Lo encontrado en esta investigación avala el supuesto que: las(os) Enfermeras(os) identifican como factores obstaculizadores el clima laboral y las relaciones interpersonales, como elementos negativos en el ejercicio del Liderazgo. Sin embargo el supuesto: evidencian con mayor propiedad factores obstaculizadores que facilitadores, no fue lo esperado, aspecto que puede servir como base para estudios multicéntricos posteriores para 
evidenciar el prejuicio que se planteó en este estudio.

Reconocen el Liderazgo en todas las funciones, siendo relevante la del ámbito asistencial, enfocado mayoritariamente a la calidad de atención, trabajo en equipo y toma de decisiones, lo que se condice que la Gestión del Cuidado representa hoy en día el rol de líder de la disciplina.

Afirman que hay un déficit en la formación profesional en Pre y Postgrado en materia de Liderazgo. Existe una ausencia de conocimiento y formación en este tema, lo que es apoyado por estudios realizados sobre el aprendizaje de esta competencia, y que genera insatisfacción en estos profesionales.

Finalmente, tal como se planteara en la introducción, el propósito de este trabajo ha sido contribuir al fortalecimiento del Liderazgo en el ejercicio de la profesión, por ende, se hace elemental reflexionar acerca de las condiciones actuales, y es un reto para la disciplina. De aquí que los resultados pueden ser considerados un llamado de alerta a profundizar esta competencia en aspectos tales como concepción, factores que intervienen, ejercicio en la profesión, formación curricular y proyección en la sociedad actual. En el mundo de hoy se espera reconceptualizar las prácticas, es decir, buscar la excelencia, la ética, proyectar la Identidad Profesional, ser Líder en la Gestión de los Cuidados.

\section{REFERENCIAS}

1. Garita M, Solís E. Práctica de Liderazgo profesional en Enfermería. Colegio de Enfermeras de Costa Rica. 2003; 25(1): 22-25.

2. Salas S, Zárate R. Informe final del Taller Internacional de Gerencia en Enfermería en los Servicios de Salud. México: Organización Panamericana de la Salud; 1999.

3. Flores H. Proyecto Tuning América Latina. Reflexiones y perspectivas para la
Educación Superior en América Latina. Informe final 2004-2007. España: Universidad de Deusto, Universidad de Groningen; 2007.

4. De Lima T, Coelho S, Adyles L, Biolchi T, Pires D, Schubert V. Influencia en los Estilos de Liderazgo del Enfermero en las relaciones interpersonales del Equipo de Enfermería. Enferm Global. 2011; 22(1): 22.

5. Tapp D, Stansfield K, Stewart J. La Autonomía en la práctica de enfermería. Aquichan. 2005; 5(1): 114-127.

6. Strapasson M, Medeiros C. Liderazgo de Cambio en Enfermería. Rev Bras Enferm. 2009; 62(2): 228-33.

7. Franca P, Cardozo A. Liderazgo: Un desafío para el enfermero recién licenciado. Rev enferm. UERJ. 2010; 18(4): 591-597.

8. Beneitone P, Esquetini C, González J, Marty M, Siufi G, Wagenaar R. Reflexiones y perspectivas de la Educación Superior en América Latina. Proyecto Tuning América Latina 2004-2007. España: Universidad de Deusto, Universidad de Groningen; 2007.

9. Marriner A, Raile M. Gestión y Dirección de Enfermería. 8a ed. España: Mosby-Elsevier; 2007.

10. Malone BL. Clinical and professional leadership. En: Hamric AB, Spross JA, Hanson $\mathrm{CM}$, editors. Advanced nursing practice. Philadelphia: WB Saunders Company; 1996.

11. Rodríguez A, Muñoz M, Marzo A, Sánchez F. Liderazgo en Grupos de Trabajo Enfermeros. Percepciones. Enferm Clin. 2011; 19(4): 206-209.

12. Zaccaro S. The Nature of executive leadership: a conceptual and empirical analysis of success. Washington D.C.: American Psychological Association; 2001.

13. Telaranta S. Administración-GestiónCalidad. Opiniones sobre Gestión y Liderazgo en Enfermería. Enferm Global. 2007; 1(1): 1-14.

14. Rodríguez V. Liderazgo de administrado- 
res de Enfermería en la toma de decisiones y la comunicación departamento de Pediatría Hospitalaria Dr. Pastor Oropeza Riera Biblioteca Lascasas [Internet]. 2009 [citado 15 agosto 2012]; 5(2). Disponible en http://www.index-f.com/lascasas/documentos/lc0416.php

15. Dos Santos I, Castro C. Características pessoais e profissionais de enfermeiros com funções administrativas atuantes em um hospital universitário. Rev Esc Enferm USP. 2010; 44(1): 154-160.

16. Miranda M. Enfermería en los Procesos de Reforma del Sector Salud. Colegio de Enfermeras de Costa Rica. [Internet]. 2001 [citado 23 abril 2011]; 24(1). Disponible en: http://www.binasss.sa.cr/revistas/enfermeria/v24n1/art8.htm

17. Diario Oficial de Chile. Autoridad Sanitaria. Ley $\mathrm{N}^{\circ} 19.937$ [Internet] 2004 [citado 23 abril 2011]. Disponible en http://www. leychile.cl/Navegar?idNorma $=221629$

18. Diario Oficial de Chile. Código Sanitario. Ley $\mathrm{N}^{\circ}$ 19.536. [Internet] 1968 [citado 23 abril 2011]. Disponible en http://www. ispch.cl/ley20285/t_activa/marco_normativo/7c/dfl_725_67.pdf

19. Disponible en Milos P, Bórquez B, Larraín A. La Gestión del Cuidado en la legislación Chilena: Interpretación y alcance. Cienc enferm. 2010; XVI(1): 17-29.

20. Milos P, Larraín A, Simonetti M. Categorización de Servicios de Enfermería. Propuesta para asegurar una atención de calidad en tiempos de escasez de enfermeras. Cienc enferm. 2009; XV(1): 17-24.

21. Lorda S. El Consentimiento Informado: teoría y práctica. Med Clin (Barc). 1993; 101(5): 174-182.

22. Glaser B, Strauss, A. The discovery of Grounded Theory. Chicago: Aldine, 1967.

23. Miles MB, Huberman A. Qualitative data analysis: an expanded sourcebook. Newbury Park, CA: Sage; 1994.

24. Van Maanen J. Qualitative Methodology. California: Sage; 1983.

25. Guba E, Lincoln Y. Naturalist Inquirí.
London: Sage; 1985.

26. Galdames L. ¿Por qué desarrollar competencias generales en los alumnos de Enfermería? Rev Enfemr. 2001; 4 (4): 14-16.

27. Jiménez G. Administración-Gestión-Calidad. Concepciones acerca del Liderazgo de Enfermería. Enferm Global. 2006; 5(9): 1-11.

28. Pardo C. Liderazgo personal e interpersonal en los coordinadores y profesionales en Enfermería que laboran en las IPS de $3^{\text {er }}$ nivel de Atención de Cartagena [tesis maestría]. [Bogotá]: Universidad Nacional de Colombia; 2011. 139 p.

29. Linton J, Farrell MJ. Nurses' perceptions of leadership in an adult intensive care unit: A phenomenology study. Intensive Crit Care Nurs. 2009; 25(2): 64-71.

30. Hernández C. Estilos de Liderazgo de enfermeras y enfermeros del Servicio Cántaro de Salud. Reduca (Enfermería, Fisioterapia y Podología). 2010; 2(1); 593-606.

31. Medingure J. El Liderazgo de Enfermería desde la perspectiva profesional, Lima, Perú. Rev Cienc Salud. 2007; 2(2); 80-89.

32. Rozo S, Abaunza M. Liderazgo transaccional y transformacional. Av. Enfer. 2010; 28(2): 107-115.

33. Burns J. Leadership. New York: Harper Colophon Books; 1978.

34. Estefo S, Paravic T. Enfermería en el Rol de Gestora de los Cuidados. Cienc enferm. 2010; XVI(3): 33-39.

35. Schebella G, Müller A, Dall'agnoll C, Cavalcanti B, Dos Santos D. Liderazgo en Enfermería: análisis en el proceso de elección de jefes. Rev Lat Am Enfermagen. 2010; 18(6); 1-9.

36. Harrison L, Ray A, Cianelli R, Rivera M, Urrutia M. Competencias en Investigación para diferentes niveles de formación de Enfermeras: una perspectiva Latinoamericana. Cienc enferm. 2005; XI(1): 5971.

37. Zárate R. La Gestión del Cuidado de Enfermería. Index Enferm. 2004; 13(44-45): 42-46. 
38. Aiken L, Clarke S, Cheung R, Sloane D, Silber J. Educational levels of hospital nurses and surgical patient mortality. JAMA. 2003; 290(12): 1617-1623.

39. Registered Nurses'Association of Ontario. Healthy Work Environments Best Practice Guidelines. Developing and Sustaining Nursing leadership [Internet]. Ontario: Registered Nurses'Association of Ontario; 2006 [actualizado 2013; 15 agosto 2012]. Disponible en: http://rnao. ca/bpg/guidelines/developing-and-sustaining-nursing-leadership

40. Walker R, Cooke M, Henderson A, Creedy DK. Characteristics of leadership that influence clinical learning: a narrative review. Nurse Educ Today. 2011; 31(8): 743-756.

41. Buchan J, Calman L. La escasez de Enfermeras Especialistas en el mundo. Suiza: Consejo Internacional de Enfermeras; 2004. 